\title{
A Link Quality Based Dispersity Routing Algorithm for Mobile Ad Hoc Networks
}

\author{
Sujatha.P. Terdal \\ Dept. of CSE,PDA College of Engineering Gulbarga,India \\ suja_pst@rediffmail.com \\ Dr.V.D.Mytri \\ Shetty Institute of Technology,Gulbarga, India \\ vdmytri@gmail.com \\ Dr. A.Damodaram \\ Dept. of CSE,JNTU college of Engineering,Hyderabad,India \\ damodarama@gmail.com
}

\begin{abstract}
Multipath routing mechanisms have been preferred over single path routing to provide parallel fail safe paths and to maximize throughput. Applying traditional shortest path metric for multipath route selection leads to traffic concentration at some nodes resulting in congestion, thereby causing performance degradation. Interference caused due to neighbouring node traffic is another factor that further limit its performance gains. Towards this, to ease out congestion and improve network resource utilization we devise a load balancing strategy that splits the load among multiple paths according to link quality. Link quality metric is formulated considering the current load condition, traffic interference and energy of the intermediate nodes. To study the effectiveness of the proposed load distribution method we compare it with another load balancing strategy that forwards traffic along one path ,found using our link quality metric and which switches over to alternate best path on route failure. Simulation results conducted show that proposed load splitting strategy is able to achieve improvement in performance in terms of reduced latency and improved throughput. Network Life time is also improved.
\end{abstract}

Index Terms - MANET, Multipath Routing, Congestion, Interference Awareness, Energy, Load balancing, Traffic Splitting

\section{INTRODUCTION}

Mobile Ad Hoc networks (MANET) play an important role in providing ubiqutous services and are envisioned to support future generation networks . Research in this area is becoming popular due to wide ranging applications supported. MANET's are characterized by fast changing topology, limited battery power and constrained resources. MANET's enable easy deployment as they do not need any infrastructure like base stations.

As MANET's are expected to support real time multimedia services, network capacity provisioning and
Quality of Service(QoS) guarantees are some of the key issues to be addressed. Multipath routing schemes are often seen as a better alternative in not only providing parallel fail safe paths but also seen as a good choice for facilitating network provisioning and realizing QoS guarantees. Its effectiveness in providing bandwidth aggregation and load balancing is already proved in the wired networks and is now currently gaining relevance in the context of Mobile Ad Hoc Networks and Mesh networks. In this work, a design for load balancing the network load which can tackle congestion and at the same time extend network life time is proposed. Multipath load balancing overcomes the capacity limitation of single path routing by distributing data traffic on to multiple paths and reducing congestion by routing traffic through lightly loaded paths.

However, the advantages of multipath routing comes at a price as concurrent data transmission along multiple paths interfere with each other. Further, when network traffic starts increasing, there will be increased level of contention among nodes coupled with higher collision level consequently leading to packet drops and network level congestion. So a routing metric needs to be judiciously selected while constructing multiple paths that avoids high interference areas and high contention areas. To ease out the network wide congestion ,we adopt congestion aware metric for route selection combined with a good traffic distribution strategy that distributes the load according to quality of a path .

As all nodes transmit on the same channel ,neighbouring nodes compete with each other for channel bandwidth resulting in inter-flow interference[1]. The inter-flow interference level experienced is directly proportional to the amount of load generated by the neighbouring nodes. It also has adverse effect on quality of the received signals significantly and can cause collisions ,packet loss leading to more energy consumption. We adopt MAC layer contention window size and interface buffer size of a node and its neighbouring nodes to capture inter-flow interference and 
load .To ensure network longevity only the intermediate nodes which have enough energy are allowed to participate in the routing process. The rest of the paper is organized as follows.

In section 2, we review related prior work. In section 3 , design of the proposed protocol is described. Section 4 introduces the working of Link Quality based Dispersity Routing Protocol .Performance evaluation and simulation results are presented in section 5 ,while conclusions are offered in section 6 .

\section{RELATED WORKS}

Quality of Service enhancements to routing protocols has become a necessary feature to meet the performance requirements of applications. Towards this, load balancing solutions are seen as an important way to alleviate congestion and for satisfying Quality of Service demands. Load balancing refers to distribution of network traffic among all portions of the network uniformly to improve resource utilization and fairness among nodes effciently. Shortest path routing does not consider traffic or load status of the intermediate nodes during route construction. This leads to increased concentration of traffic along the links which are part of the shortest path resulting in packet loss and faster energy drain rate. Load aware or delay based routing circumvents congested routes and select routes with lesser traffic or delay. Load or delay aware routing metrics [2],[3],[4],[5],[6] have been adopted by single path routing protocols to avoid congested routes.

Multipath routing is a natural choice for distributing the load evenly across the network. One important component of load balancing is that of traffic distribution. There are two strategies for distibuting traffic using multiple paths[7]. In one load balancing strategy ,multipath routing protocol may decide to forward the traffic over only one lightly loaded path and keep others as backup paths[8],[9] and in other case, traffic may be distributed over multiple paths concurrently[10],[11]. Load balancing protocols adopt metrics that characterize existing load or traffic on the paths and try to avoid such congested areas. Hence, routing paths discovered by load balancing protocols are always longer than shortest hop metric based routing protocols. One of the earliest works, MSR[10] introduces load balancing which distributes traffic using weighted round robin scheduling strategy. The criterion used to distribute the load is based on round trip time or RTT which is not an effective method of load evaluation. [11] distributes load such that total number of congested packets on each path is equal and does load splitting at packet level granularity. AMOR[12] chooses ETX (Expected Transmission Count) as the metric to optimize upon load distribution instead of shortest hop metric. [13] proposes a solution for load balancing, where every intermediate node records the number of primary paths passing through it. During route discovery, RREQ packets are dropped if the number of primary paths passing through that node has reached a maximum limit.
[14] considers number of valid routes of the intermediate nodes to represent load state and the source distributes the load based on the capacity of each path. In order to predict the occurrence of congestion in future, count of RREP packets is used in[15]. Then a combined cost metric using current and future congestion status is used to evaluate the load and accordingly balance the load. [16] uses number of retransmission attempts for a successful data transmission and it is taken as a function of energy expended for transmission and reception. It addresses the issue of energy balancing but not balancing of the load.

But the above load balancing mechanisms does not consider the effect of interpath interference arising due to concurrent data transmissions. Intereference is a limiting factor in ad hoc networks that greatly influences the network's performance due to the shared nature of the medium. The impact of interference has been studied [17] by presenting a multi-commodity flow problem with interference related constraints using a conflict graph to model the constraints imposed by interference. It is shown that adopting interference reduction measures can bring in improvement in the throughput. [18] presents a stochastic framework for analysis of interference and develops a mathematical model to assess network capacity for multipath routing using non linear programming. But these methods proves to be inefficient even for small networks due its complexity .[19] tries to reduce the transmission range of the nodes using power control to minimize unnecessary interferences experienced by the neighbouring nodes .[20] uses topology control to reduce the effect of interference . [21] proposes an interference aware metric Network Allocation Vector Count (NAVC) based upon which delay and available bandwidth is predicted .

As there are different approaches used to consider interference, proposed work adopts a simple traffic based approach to measure interference. This paper adopts a load balancing solution with interference awareness. The solution proposed here, estimates the load as well as interference based on channel condition indicators like contention window and MAC Layer buffer size.

\section{PROTOCOL DESIGN}

In this section, we deal with the design of proposed work Link Quality based Dispersity routing algorithm for load balancing(LQLB) .

\section{A. Problem Formulation}

The goal of this design is to find lightly loaded node disjoint paths with less interference and which balances the traffic over multiple paths adaptively according to the link conditions and at the same time extend the network life time. Key issue to be addressed while splitting traffic over multiple paths is ,how to apportion the traffic according to the quality of each path. Next, is the choice of an appropriate metric that reflects quality of a path.

Before we introduce our design, we will putforth the working principle behind it. Consider a sample network 
shown in figure 1 . Node $A$ wants to communicate with node $H$. Nodes $B, C, D, E, F, G$ are intermediate nodes. Filled circles are neighbor nodes . Adjacent rectangles beside the neighbor nodes show the size of MAC layer buffer. Shaded portion of the rectangle indicate unutilized portion of the queue. Node disjoint multipath routing algorithm would select three paths $A B C H, A D E H, A F G H$ based on shortest hop metric without considering the quality of the links. But selecting path $A B C H$ would lead to congested links and severe throughput degradation due to more number of neighbours actively transmitting resulting in more amount of contentions . Bigger queue size of a node would mean higher load and intense traffic activity. Comparatively, path $A F G H$ would experience lesser level of contention as it has only one neighbour with less load. Our protocol will not select path $A B C H$ as we consider contentions caused due to neighbouring node activity. Our route selection procedure not only finds node disjoint paths but also integrates contention window size and queue size calculation to capture interflow interference. It also balances load across paths by traffic splitting .

In the following section, we describe how this proposed metric link-Quality accounts for interference caused due to neighboring node traffic and also measures the current load of a link.

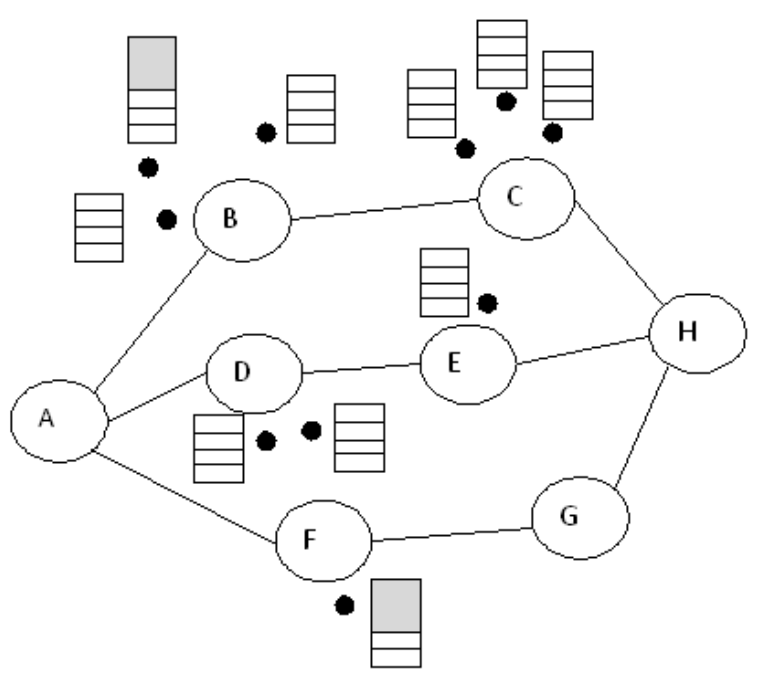

Figure 1 : Sample Network depicting interfering neighbours and their queue stataus.

\section{B. Metric for Interflow Interference and Load}

One of the important factors that cause interference is traffic generated by the nodes. Traffic produced per node and the traffic relayed by the node determines the amount of interference created [22]. In addition ,number of neighbour nodes within the transmission range and interference range are potential sources of interference. Level of interference experienced by a node also depends on distance of its neighbour nodes. Active neighbours busy transmitting is another factor influencing interference. Ad hoc networks uses DCF[23] as the medium access mechanism supported by IEEE 802.11 MAC layer protocol. When one or more nodes try to access the channel simultaneously collision is experienced following which contention window is set to $\mathrm{CW}_{\text {min. }}$. The size of the contention window determines how long a node would backoff before attempting to gain access to the channel. In other words CW[5] indicates the busyness of the medium and can be considered as a useful metric for contention and traffic interference. A large CW would mean more amount of traffic interference. As nodes transmit using the same channel, they have to contend with each other for sharing bandwidth causing traffic interference [1]. The level of interference depends on the amount of load generated by the interfering nodes.

In order to capture the effects of neighboring node transmissions we use average size of contention window and the queue size of the current node and its neighboring nodes. Traffic conditions of the neighboring nodes is propagated using Hello packets periodically. Each node maintains a neighbor table shown below to store traffic information of its neighboring nodes.

\section{LINK QUALITY BASED DISPERSITY ROUTING PROTOCOL}

This section describes a solution for load splitting derived by integrating path quality metric into AODVM[24] routing protocol.

\section{A. The Load Splitting algorithm}

During initialization phase every node collects information about the size of its $\mathrm{CW}$, its buffer occupancy level, and remaining battery level. In order to contain transient bursts the size of contention window found is averaged out using exponential weighted moving average method. Similarly average size of the interface buffer and energy values are calculated. Each node then constructs a neighbour table which stores the list of its neighbouring nodes along with their respective traffic load which is again expressed by its contention window size. AODV's Hello messages are modified to convey information about neighbour node's contention window size ,queue size and battery level. Using the information from the Hello messages each node constructs a neighbour table. Format of a neighbour table is shown below.

Table 1. Neighbor Table contents

\begin{tabular}{|c|}
\hline TimeStamp \\
\hline Avg-CW $_{\text {size }}$ \\
\hline Avg-Queue $_{\text {size }}$ \\
\hline Rem Energy Level \\
\hline Node-Id \\
\hline
\end{tabular}

Using the information from the neighbour table ,each intermediate node stores the cost found using the 
equation 1 where $i$ refers to an intermediate node ,n refers to number of neighbor nodes of $i$.

$$
\operatorname{Cost}_{i}=C W_{i} * Q-\text { Size }_{i} * 1 / \text { Rem-Energy }+\sum_{\mathrm{j}=0}^{\mathrm{n}}\left(C W_{j} * Q-\text { Size }_{j}\right)
$$

$C W$ indicates average size of the contention window, $Q$ Size represents the average size of the MAC Buffer. Quality of a path is taken as the cumulative cost of intermediate nodes that form a path. We enhance AODVM's route discovery procedure to support link quality based load splitting. Route Request packets are overloaded to note the size of contention window, remaining energy level and queue size information by the intermediate nodes. Let $\operatorname{cost}_{A}$ be the cost of path $A$, $\operatorname{cost}_{B}$ the cost of path $B$ and $N$ be the total amount of data to be forwarded from a source to a particular destination . Let the total cost of two paths $A$ and $B$ be Total-cost, then the number of data packets $\left(N_{A}\right)$ forwarded along the first path $A$ would be

$$
N_{A}=\left(\operatorname{cost}_{A} / \text { Total- } \cos t\right) * N
$$

\section{B. Route Discovery}

Route Discovery procedure is initiated by the source when it does not have a route in its cache to a destination by broadcasting a RREQ packet. When an intermediate node receives a RREQ packet it forwards the packet only if the node has energy beyond a threshold value. While a RREQ is forwarded ,each intermediate node inserts its cost which is computed as in (1). Similarly every internediate node checks if it has enough energy and subsequently appends its cost in to the RREQ packet . When the RREQ reaches the destination it will have the cumulative cost of the path. When additional RREQ's arrive destination selects four least cost paths. Destination then generates RREP for the four selected best paths. Source, after receiving the best paths starts with data transmission by distributing the packets proportional to the quality of each path found as in (2). Only two paths with best cost is selected for data transmission.

\section{Route Maintenance}

Route Maintenance is initiated when a link break is detected by a node by transmitting a RERR packet . Data transmission is continued along the next prediscovered path stored by the source. Number of packets forwarded along this new path is proportional to its cost . If all prediscovered paths fail a new route discovery procedure is initiated by the source.

\section{PERFormanCE Evaluation}

In this section, we bring out a comparative study of two load balancing strategies. First one being $L Q L B$ 1path, a load balancing strategy that distributes the load along one best path computed by the proposed link quality metric. Source switches to next best path when currently active path fails due to link failure. Second load balancing strategy $L Q L B-2 p a t h$ distributes the traffic along two best paths concurrently .

\section{A. Simulation Environment}

Evaluation of the proposed protocol is carried out using OMNET++, a discrete event simulator which supports complete physical, data link and MAC layer models for simulating wireless ad hoc networks. We simulated a network of 50 mobile nodes placed randomly in an area of $1500 \times 600$ square meters. Each node is assumed to use IEEE $802.11 \mathrm{~b}$ radio interface. A source and a destination is selected randomly. Free space propagation model is assumed as the channel model. Each node is assumed to have a constant transmission range of 250 meters and a channel capacity of $2 \mathrm{Mbps}$. Source destination pairs are spread randomly over the network. Mobility pattern of the mobile nodes is generated using Random waypoint model . Speed of a mobile node is assigned as 5 meters/sec. Energy level of all nodes is initialized as $100 \mathrm{~mJ}$. Amount of energy expended while transmitting and receiving is assumed to be $0.0003 \mathrm{~mJ}$ and $0.0001 \mathrm{~mJ}$ respectively.

We evaluate the performance of the proposed work using following parameters.

1. Packet Delivery Ratio(PDR): This is defined as ratio of number of packets that have successfully reached the destination to the total number of packets sent by the source. This metric is expressed in percentage

2. Throughput: Throughput refers to the total number of packets successfully reaching the destination per second. This metric is an indicator of the quality of a routing path and the effectiveness of the load balancing mechanism.

3. Average contention window size: The size of the contention window reflects the level of contention suffered by a node. A large contention window indicates larger level of traffic interference. It is measured in terms of number of slot times.

4. Route Life Time: Route Life Time indicates how stable a path is and it shows how long can it sustain data transmission. It is measured by the time elapsed between the generation of a RREP packet and the generation of a RERR packet .

5. MAC Buffer overflow: This occurs when a node cannot enqueue a packet as it's queue is full due to congestion. In other words, low buffer overflow level indicates how well traffic is distributed over the entire network.

6. Average Energy Consumed per Bit: Remaining energy of the network is sum of the remaining energy of all nodes in the network. Similarly initial energy of the network is found by summing up initial energy levels of the nodes. Energy consumed then is the difference between the remaining energy and the initial energy of the network . Ratio of the energy consumed to total number of bits transferred gives the average energy consumed per bit.

7. Network lifetime :A mobile node with its remaining energy level less than a threshold equal to $20 \%$ of the initial energy is considered dead. Network lifetime is 
equal to the difference in time when network is initialized to the time when the first node is dead.

\section{B. Simulation results}

Performance of two load balancing strategies $L Q L B$ 2 Paths , LQLB-1Path is compared. Figure 2 shows the packet delivery ratio achieved for both strategies. $L Q L B$ 2 Paths performs better than LQLB-1Path.This shows the advantage of performing load balancing using traffic distribution along two paths. Initially there is not much difference between two schemes ,but as network load increases beyond $100 \mathrm{Kbps}$ there is marked difference in their performance asserting the fact that load distribution is advantageous when network traffic is high.

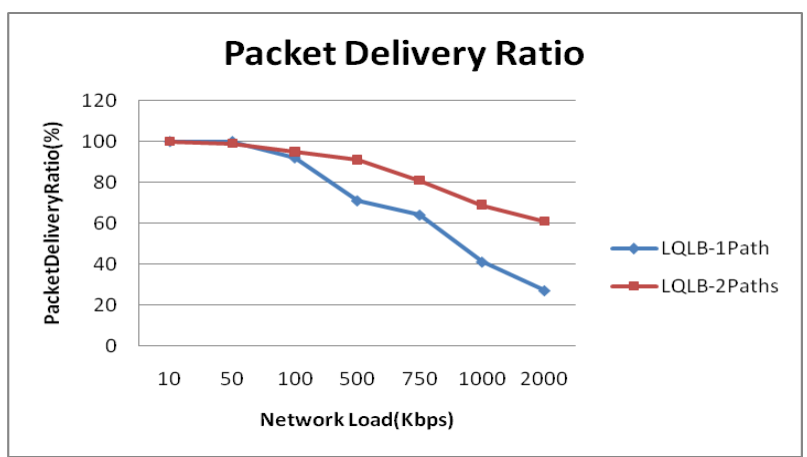

Figure 2: Packet Delivery Ratio under varying Network Load.

Increase in the PDR achieved initially is around $20 \%$ but when network load increases ,up to $55 \%$ improvement is achieved. On an average, improvement in the PDR achieved is $27 \%$. Another parameter of interest studied is throughput.

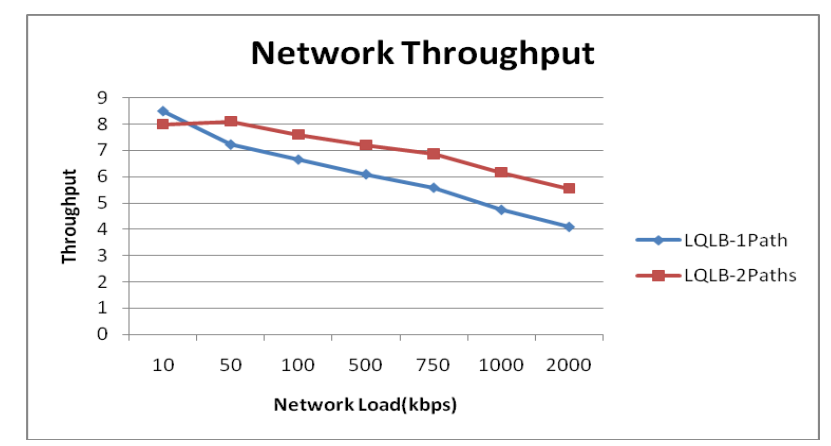

Figure 3:Throughput against varying Network Load.

As this proposed work avoids high contention areas and selects routes with less interference, packet loss and retransmissions will be reduced. This accounts for the increase in throughput which can be observed from the Fig. 3. The size of the contention window indicates backoff interval of a node on the occurrence of collision. Lesser size would mean lesser level of contention and hence less traffic interference.

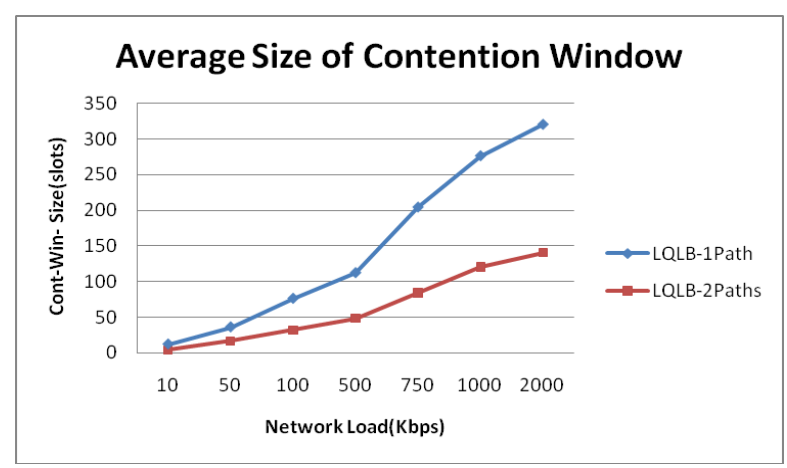

Figure 4: Contentention Window Size against varying Network Load.

Average growth of contention window is lesser in case of $L Q L B-2$ Paths. This can be attributed to the fact that our protocol avoids routes which will have less neighboring node contention. In other words $L Q L B$ 2Paths avoids nodes with lesser level of traffic activity at the neighboring nodes. Fig. 4 shows this.

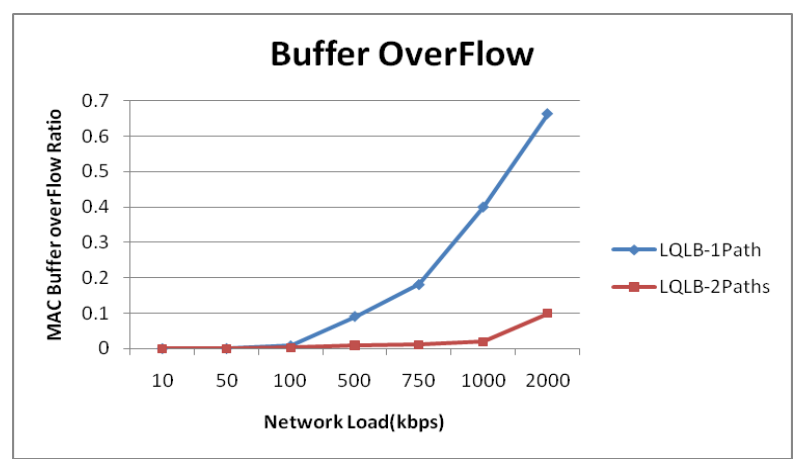

Figure 5: MAC Buffer overflow under varying Network Load.

To further demonstrate the advantage of our protocol we compare the level of buffer overflow .Buffer overflow occurs when nodes gets congested. Amount of buffer overflow in $L Q L B-2$ Paths is very less compared to $L Q L B$-1Path method that does not split the load which is shown in Fig. 5. Queue size monotonously increases as the node is depreived of channel due to contention by neighboring node traffic. As this proposed protocol can distribute the load evenly lesser amount of buffer overflow occurs at the nodes in LQLB-2Paths case .

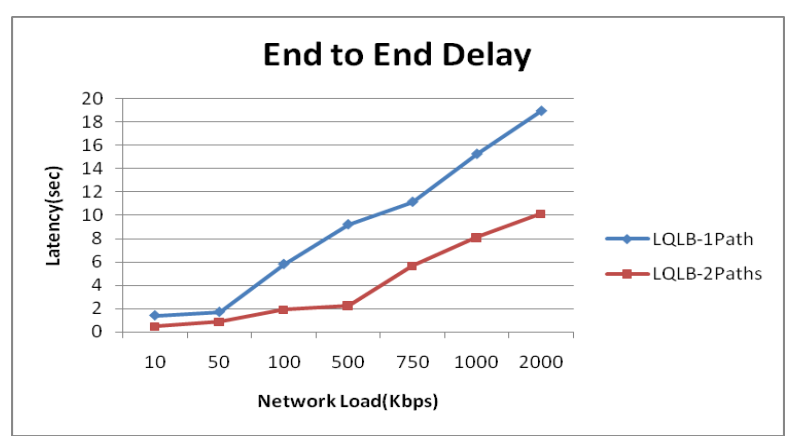

Figure 6: Latency against varying network load

Data packets are simultaneously routed along two paths because of which latency tends to decrease . 


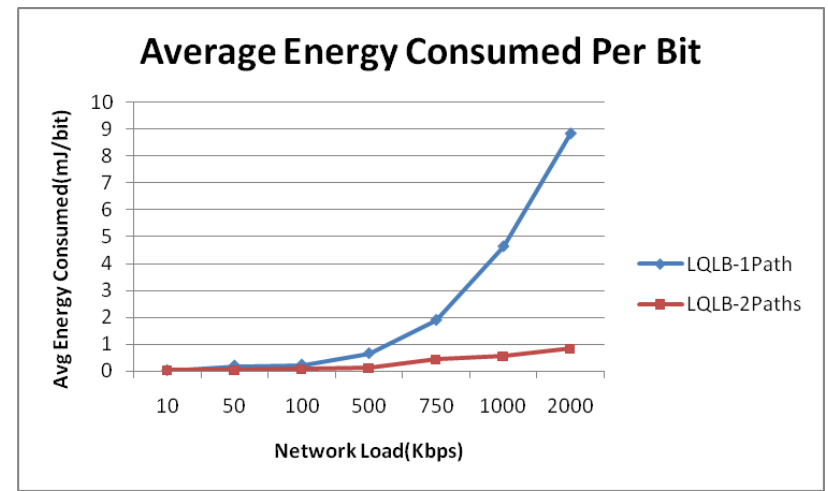

Figure 7: Average Energy Consumed against varying network load

But this method has the disadvantage that it gives rise to additional overhead at the transport layer in the form of packet reordering. Fig. 6 shows the improvement in the latency achieved. Other than reducing end to end delay, load balancing mechanisms positively influence the energy consumption also. Adopting proper load balancing solutions can reduce creation of hot spots which are highly congested regions.

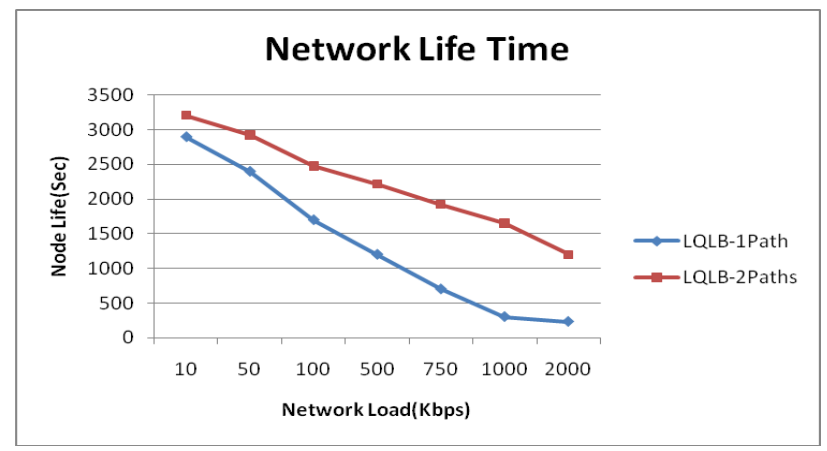

Figure 8: Network Life Time against different Network Load

Nodes in congested regions tend to loose energy at a rapid rate due to increased number of retransmissions resulting out of packet losses. Energy expenditure incurred by adopting load balancing is shown in Fig. 7. Energy expended is less in case of load balancing with traffic spliiting as $L Q L B-2$ Paths have lesser degree of control overhead thereby saving upon the transmission/retransmission cost . As energy expended by the nodes gets reduced due to traffic splitting, network lifetime is extended. Fig 8 and 9 show the enhancement achieved.

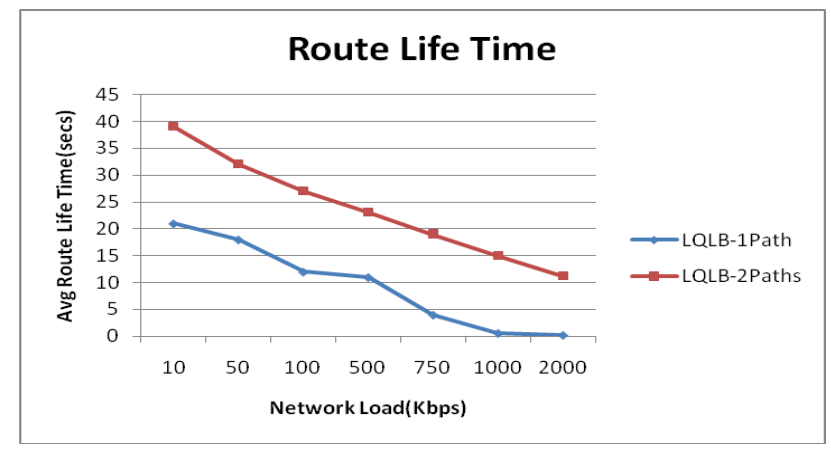

Figure 9: RouteLife Time against varying Network Load
Further set of simulations were conducted to evaluate the performance against mobility .

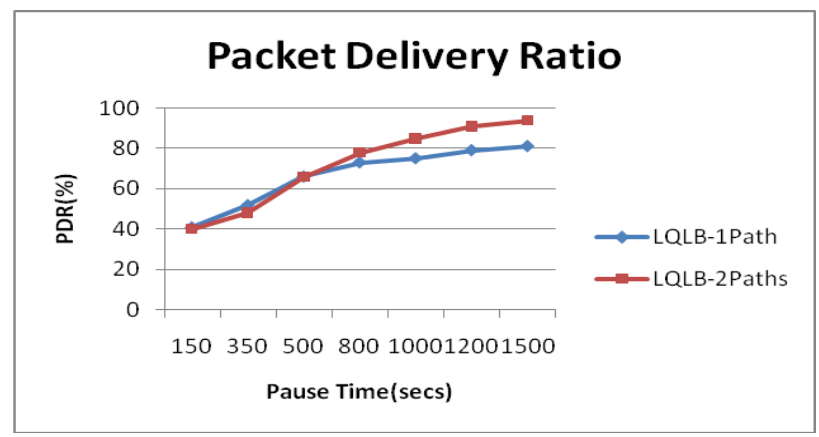

Figure10 : Packet Delivery Ratio for different pause Times.

For this pause time is varied keeping the node speed $5 \mathrm{~m} / \mathrm{s}$. Though initially both the methods of load balancing show similar performance, as data transfer progresses load splitting mechanism outperforms which is shown in Fig. 10.

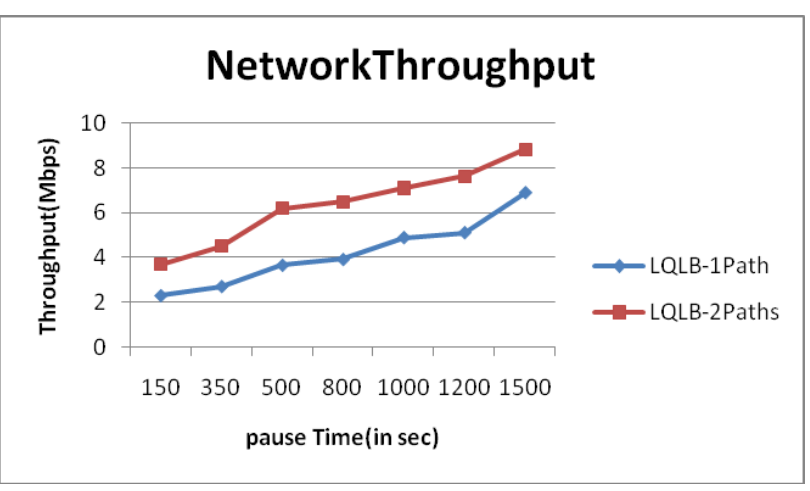

Figure 11: Network Throughput against different pause Times.

Throughput is also compared for both load balancing mechansims. Load splitting aimed at balancing the load brings improvement in throughput. Fig. 11 shows the increase in throughput experienced which increases when node mobility decreases as there will be fewer route changes.

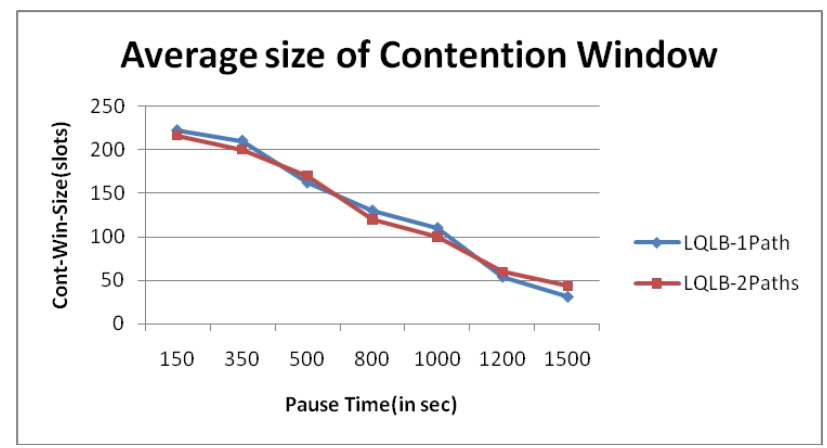

Figure 12: Contention Window variation for different pause Times.

Contention window size does not show much difference for both schemes as network load is not varied Fig. 12 shows the contention level experienced by the nodes which is affected only when traffic level varies. 


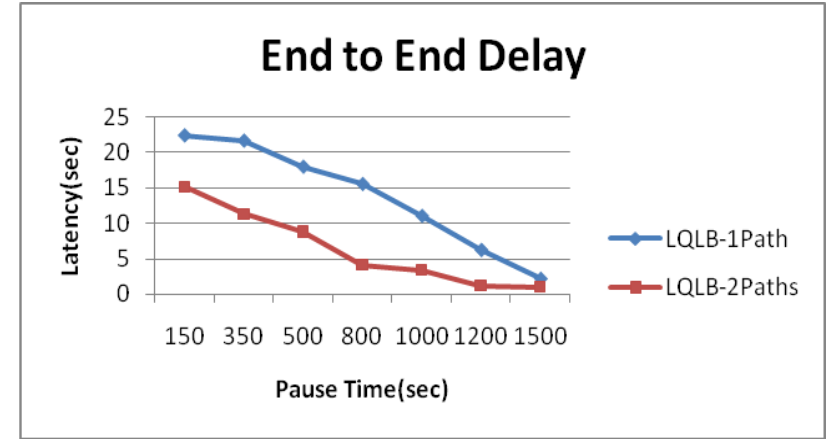

Figure 13: Latency for different pause Times.

Latency also shows improvement when the degree of mobility is varied. Load splitting based on path quality has reduced latency under reduced level of mobilty. For increased level of mobility latency invariably increases . When mobility level is very low or when network topology is almost static there is no difference in latency for both schemes. Fig. 13 depicts the latency experienced by both the schemes. Under increased level of mobility energy consumed in $L Q L B-2$ Paths is lesser than $L Q L B$ 1Path load balancing strategy. Energy expended by the nodes is reduced as $L Q L B-2$ Paths selects routing paths based on path quality and hence will not route through congested areas and high interference regions. Fig. 14 shows this.

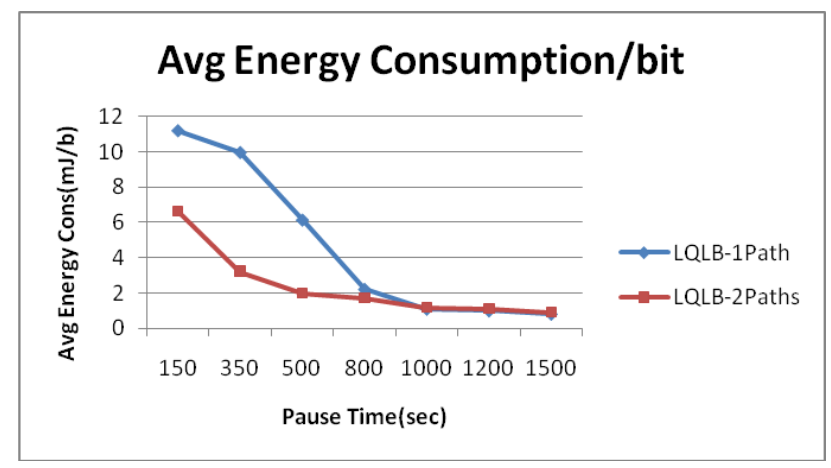

Figure 14: Energy consumption against pause Time.

A network sustains for a longer period of time if the rate at which energy is consumed for data transmision and reception activities is less. As LQLB-2Paths will have lesser amount of packet losses and retransmissions ,Network lifetime is better. Fig. 15 shows Network Life Time achieved for both schemes .

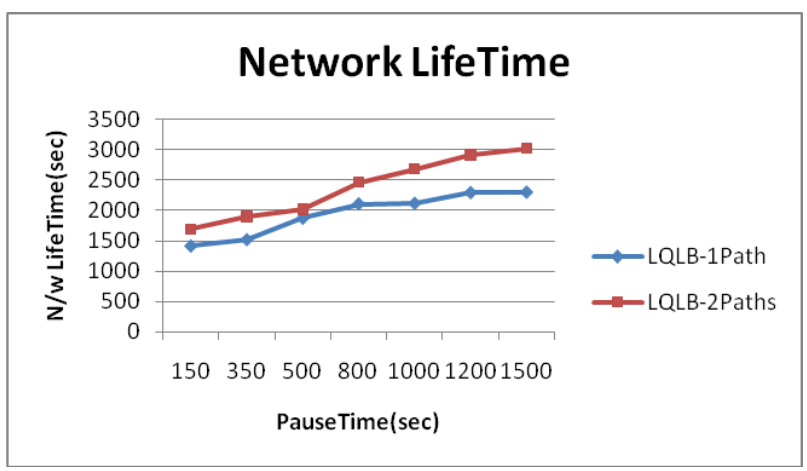

Figure15: Network life Time against pause Times.
Link breaks leading to generation of RERR packets are used to asses route lifetime . Path quality based routing enhances route life time. Link breaks are caused due to congestion or mobility.

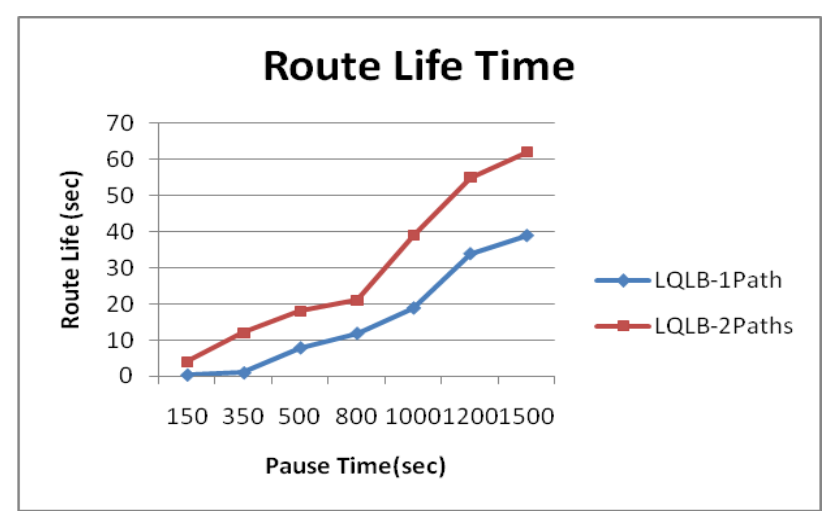

Figure 16: Life Time of a route for different pause Times.

Fig.16 shows improvement in route life time for $L Q L B-2$ Paths when compared to LQLB-1Path due to the reduced amount of control overhead .

\section{CONCLUSIONS}

A fundamental issue that adversely impacts the performance of multipath routing protocol is that of interference. Another deterrent factor is that of link level congestion. Hence an efficient load balancing solution is necessary that addresses both the above mentioned issues. This paper presents an algorithm that leverages link quality information for load balancing by distributing the load . A metric that effectively captures interflow interference and that also accounts for the traffic load is proposed. Link quality is evaluated by considering contention, load and energy metrics. Interfering traffic load due to neighbouring nodes is also considered. Performance evaluation of the proposed traffic splitting strategy is carried out under varying network load conditions and mobility levels show improvement over the load balancing strategy without traffic splitting.

\section{REFERENCES}

[1]Devu Manikantan Shila ,Tricha Anjali ,'Load Aware Traffic Engineering for Mesh Networks", Computer Communications(31) 2008 Elsevier ,pp 1460-1469.

[2]H. Hassanein and A. Zhou, "Routing with Load Balancing in Wireless Ad Hoc Networks", in Proc. ACM MSWIM, Rome, Italy, July (2001).

[3]Yang Li, Hong Man "Three Load Metrics for Routing in Ad Hoc Networks "Proc. of Vehicular Technology Conference, September 2004:2764-2768,IEEE 2008.

[4]Lijuan Cao Teresa Dahlberg "Path Cost Metrics for Multi-Hop Network Routing” , IEEE ,pp 1521(2006).

[5]X.M. Gao, X.M. Zhang, D. Shi, F.F. Zou, W.B. Zhu, "Contention and Queue-aware routing protocol for mobile ad hoc networks", Proc. of the 3rd IEEE 
International Conference on wireless Communications, Networking and Mobil Computing (WICOM'07), Sep. 2007.

[6]F. F. Zou, X.M. Zhang, X.M. Gao, D. Shi, E.B. Wang, "Load balance routing using packet success rate for mobile ad hoc Networks", Proc. Of the 3rd IEEE International Conference on ICOM'07), September. 2007.

[7]Jack Tsai, Tim Moors, "A Review of Multipath Routing Protocols : From Wireless Ad Hoc to Mesh Networks", Proceedings of ACoRN Early Career Researcher Workshop on Wireless Multihop Networking,Jul 17-18, 2006.

[8]Sujatha.P.Terdal, V.D.Mytri, A.Damodaram, "Load Aware Routing Mechanism for improving Energy Efficiency in Mobile Ad hoc Networks" ,International Journal of Computer Applications "Vol 10, No 3 ,November 2010, pp 612.

[9]Sujatha.P.Terdal, V.D.Mytri, A.Damodaram, "Multiple Metrics based Load balancing Routing Protocol for Mobile Ad hoc Networks" ,Proccedings of AH-ICI Kathmandu ,IEEE, November 2009.

[10]Lei Wang et al, “Adaptive Multipath Source Routing in Ad hoc Networks", IEEE (2001)

[11]Pham, P. and Perreau, S., "Multi-Path Routing Protocol with Load Balancing Policy in Mobile Ad Hoc Routing," IEEE, pp. 48-52 (2002).

[12]W.Mateen, S. Raza, Z. A. Uzmi and S. Baqai, "Adaptive Multipath On-Demand Routing in Mobile Ad Hoc Networks",Lahore University of Management Sciences, Lahore 54792, Pakistan ,2005.

[13]T Murakami, I Sasase , M Bandai “ Split Multipath Routing with Load Balancing Policy (SMR-LB) to Improve TCP Performance in Mobile Ad Hoc Networks ", Proceedings of IEEE $16^{\text {th }}$ International Symposium on Personal ,Indoor and Mobile Radio Communications 2005.

[14]Lu Xuechao ,Xu Chunxiu et.al, “ Design and Reliazation of a Novel Multi-path Load-Balancing Routing Protocol in Ad Hoc Network ",Proceedings of WASE International Conference on Information Engineering ,IEEE, 2009.

[15]Zhang XiangBo ,Ki-Il Kim ,'Load-Aware Metric for Efficient Balancing on Multipath DSR Protocol in Mobile Ad Hoc Networks", Proceedings of International Conference on Advanced Technologies for Communications, IEEE, 2008.

[16]Meng Li, Lin Zhang et al," An Energy-Aware Multipath Routing Protocol for Mobile Ad Hoc Networks", ACM Sigcomm Asia 2005.

[17]K.Jain, J. Padhye, V. N. Padmanabhan, and L. Qiu, "Impact of interference on multi-hop wireless network performance," in Proc. of the ACM MOBICOM, San Diego, CA, Sept. 2003.

[18]R. de Haan, R.J. Boucherie, and J.-K. van Ommeren, " The Impact of Interference on Optimal Multipath Routing in Ad Hoc Networks “, ITC 2007, LCNS 4516, pp 803-815. Springer-Verilag.
[19]H.Tan, W.Seah, ,Dynamic Topology Control to reduce interference in MANET's", Proc of Second International Conference on Mobile Computing and Ubiquitous Networking ,2005.

[20]T. Johansson, and L. Carr-Motyckova, "Reducing Interference in ad Hoc Networks through Topology control" , Proceedings. Of the ACM/SIGMOBILE workshop on foundations of mobile computing ,2005.

[21]Liran Ma, Qian Zhang, Fengguang An, and Xiuzhen Cheng, DIAR: Dynamic Interference Aware Routing Protocol for IEEE 802.11-Based Mobile Ad Hoc Networks “,LCNS 3794, pp 508-517, Springer Verlag 2005.

[22]R.Hekmat and P. Van Mieghem ,'Interference in Wireless Multi-hop Ad-hoc Networks and its effect on Network Capacity “, Wireless Networks, 10,389399, Kluwer Academic publishers ,2004.

[23]The working Group for WLAN Standards.IEEE 802.11 Standards Part II: Wireless Medium Access Control (MAC) and Physical Layer (PHY) Specifications . Technical Report, IEEE ,1999.

[24]Z.Ye ,S.V.Krishnamurthy, S.K.Tripathi, "Framework for Reliable Routing in Mobile Ad Hoc Networks “, IEEE . INFOCOM ,2003.

[25]Andras Varga OMNET ++ ,www.omnetpp.org

Sujatha.P.Terdal is working as Associate Professor in the Computer Science and Engineering Department ,P.D.A College of Engg, Gulbarga,Karnataka. She received her M.Tech.degree from Visveshwariah Technological University ,Belgaum, India, in 2002. Currently, she is pursuing her research at Jawaharlal Nehru Technical University,Hyderabad ,India . Her fields of interest are Mobile Ad Hoc Networks and wireless networks.

Dr.V.D.Mytri Completed B.E from Karnataka University, Dharwad,Karnataka,India in 1972. He Completed his M.Tech from IIT Madras,India in 1980. He Completed his Ph.D from IISc,Bengalore,India in 1988. Currently he is working as Principal ,Shetty Institute of Technology ,Gulbarga, India. His area of interests are Digital Communication, Digital Signal Processing, Fibre Optics \& Local Networks, Multimedia/Digital Databases User Interface Technologies and Wireless Sensors Networks.

Dr. Avula Damodaram joined as faculty of Computer Science \& Engineering at JNTU, Hyderabad in the year 1989. In his over 2 decades of dedicated service. Dr. Damodaram performed distinguished services to the University as a Professor, Head of the Department, Vice Principal, Director of UGC-Academic Staff College, Director, School of Continuing \& Distance Education and Director, University Academic Audit Cell. Dr. Damodaram has successfully guided 10 Ph.D. and 2 MS Scholars apart from myriad M. Tech projects. He is 
currently guiding 9 scholars for $\mathrm{Ph}$. D and 1 scholar for MS. Dr. Damodaram is on the editorial board of 2 International Journals and a number of Course materials.

He successfully executed an AICTE research project at a cost of 7 Lakhs. Dr. Damodaram has been a UGC nominee for a number of expert and advisory committees of various Indian Universities. He has been associated with conduct of many entrance tests in the state such as ECET and ICET. Dr. Damodaram has been a Life Member, Vice-President, Director and President of a number of core committees spread all over the country. Dr. Damodaram has served the interests of the College and University teachers at the University, State and National levels. He has organized as many as 30 Workshops, Short Term Courses and other Refresher and Orientation programmes. Dr. Damodaram has published 45 well researched papers in national and International journals. He has also presented 59 papers at different National and International conferences. Dr. Damodaram visited the Universities of United States of America, Austria and the United Kingdom for presenting papers at International conferences. On the basis of his scholarly achievements and other multifarious services, Dr. Damodaram was honoured with the award of DISTINGUISHED ACADAMICIAN by Pentagram Research Centre, India, in January 2010. 